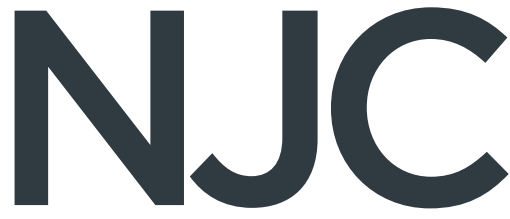

A journal for new directions in chemistry

New Journal of Chemistry rsc.li/njc

Volume 45

Number 41

7 November 2021

Pages 19167-19606
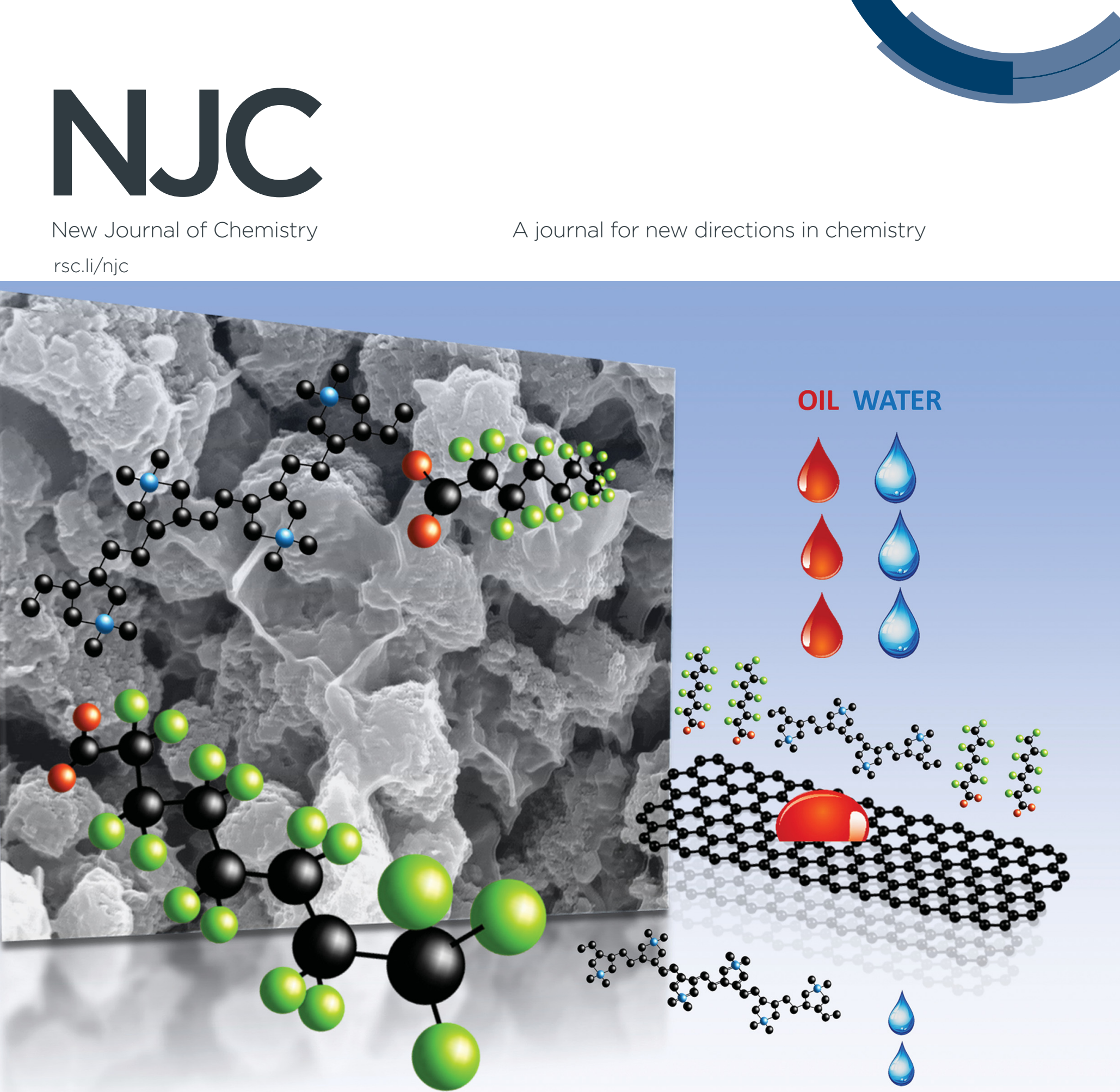

ISSN 1144-0546

ROYAL SOCIETY OF CHEMISTRY
Oleophobic composite films based on multi-layer graphitic scaffolding 
Check for updates

Cite this: New J. Chem., 2021, 45,19210

Received 3rd June 2021 Accepted 8th August 2021

DOI: 10.1039/d1nj02727a

rsc.li/njc

\section{Oleophobic composite films based on multi-layer graphitic scaffolding $\dagger$}

\author{
Rachel L. McLaren, (D) a Rosenildo C. da Costa, (D) a Christian J. Laycock, (D) ${ }^{a}$ \\ David J. Morgan, (D) ${ }^{\text {bc }}$ Michael E. A. Warwick (D) ${ }^{d}$ and Gareth R. Owen (D)*a
}

\begin{abstract}
A new oleophobic composite material synthesised by utilising plasmaexfoliated multi-layered graphitic (MLG) material as scaffolding is presented herein. The composite consisted of a polyelectrolyte/ fluorosurfactant complex derived from polydiallyldimethylammonium chloride (PDDA) and sodium perfluorooctanoate (PFO) and was used to prepare free-standing MLG composite films.
\end{abstract}

\section{Introduction}

The development of materials which possess oil/water separation capabilities represents an area of intense interest. ${ }^{1-5}$ As part of our ongoing investigations ${ }^{6,7}$ focusing on commercially obtained plasma-synthesised multi-layer graphitic material (MLG), ${ }^{8}$ we became interested in utilising MLG as a scaffold for the development of composite materials. Similar materials based on graphene oxide and $\mathrm{SiO}_{2}$ nanoparticles have previously found promise within this application. ${ }^{9}$ It was envisaged that it would be possible to develop membrane-based materials that would enable the facile separation of oil from water. Such a material could find application, for example, within the context of aircraft tanks where tanks often suffer from an ingress of water through openings.

Fluorosurfactants in particular have been heavily utilised for the fabrication of oleophobic/hydrophilic surfaces. ${ }^{1,10-20}$ These contain a sufficiently small dispersive component and a large polar component associated with their surface tension values,

${ }^{a}$ School of Applied Science, University of South Wales, Treforest, CF37 4AT, UK. E-mail: gareth.owen@southwales.ac.uk

${ }^{b}$ Cardiff Catalysis Institute, School of Chemistry, Cardiff University, Cardiff, CF10 3AT, UK

${ }^{c}$ Harwell XPS, EPSRC National Research Facility for X-Ray Photoelectron Spectroscopy, Didcot, Oxon, OX11 OFA, UK

${ }^{d}$ Energy Safety Research Institute, Swansea University Bay Campus, Fabian Way, Swansea SA1 8EN, UK

$\dagger$ Electronic supplementary information (ESI) available: Containing details of film preparation, XPS data, contact angle images and further SEM images of MLG-PP films. See DOI: 10.1039/d1nj02727a such that simultaneous oleophobic/hydrophilic behaviour is exhibited. ${ }^{21-23}$ A combination of polydiallyldimethylammonium chloride (PDDA) and sodium perfluorooctanoate fluorosurfactant (PFO) with $\mathrm{SiO}_{2}$ nanoparticles has been commonly implemented within several other research groups. ${ }^{14,18,19}$ Furthermore, Yoon and co-workers also utilised a graphene plug which enabled purification of water after its separation from hexadecane. ${ }^{18}$ In these recent studies, it has been shown that the addition of $\mathrm{SiO}_{2}$ nanoparticles provides the necessary roughness component for the amplification of oleophobic/ hydrophilic behaviour due to an increased surface area. ${ }^{20,24-27}$

In light of the success of these investigations, we synthesised a novel MLG composite containing a polyelectrolyte/surfactant complex within the context of oleophobic/hydrophilic membranes. Highly flexible and non-brittle freestanding films were fabricated which exhibit oleophobic behaviour to silicone oil, hexadecane and kerosene (Jet A-1 fuel). Herein, we wish to report a preliminary account of our investigation demonstrating that these are effective in providing oil-water separation.

\section{Results and discussion}

Synthesis and analysis of MLG-PP composite films

The first stage of our investigation involved the preparation of a MLG composite (MLG-PP) consisting of MLG, PDDA and PFO which displayed oleophobic properties. The MLG material used for this was synthesised via a plasma exfoliation process providing multi-layered stacks of graphitic material (see experimental section and ref. 6 for further details). The synthesis of the composite was achieved via a two-step process comprised of initial adsorption of PDDA onto MLG. ${ }^{18}$ This was followed by addition of PFO which led to the PDDA/PFO complexation on the surface of the MLG material, as shown in Fig. 1. The preparation of the non-covalently bound composite, MLG-PP, gave the product as a thick black gummy material, as shown in Fig. 1 (inset image). It was found that redispersion of the MLG-PP composite into methanol allowed us to produce 

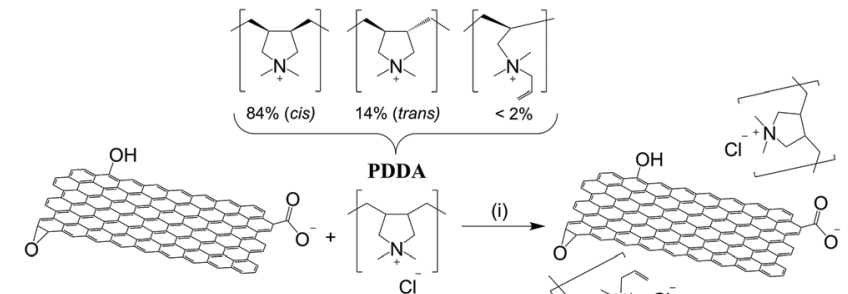
$\mathrm{N}^{+} \mathrm{Cl}^{-} \mathrm{MLG} / \mathrm{PDDA}$

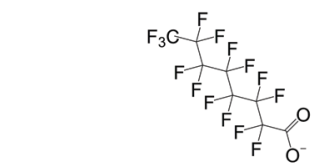

MLG-PP Composite
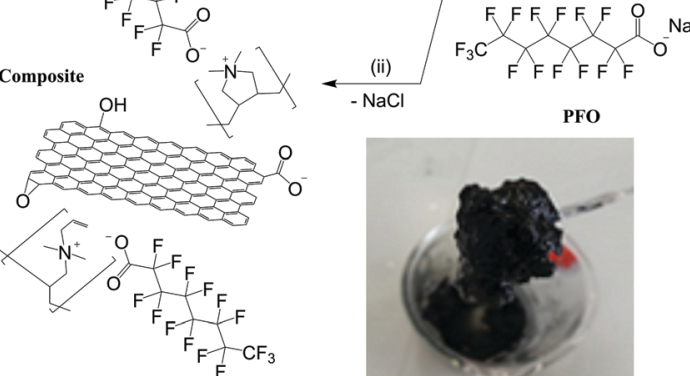

PFO

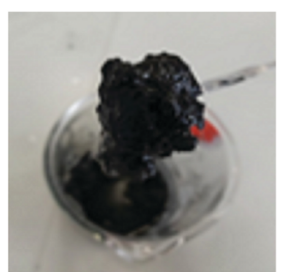

Fig. 1 Synthesis of MLG-PP composite via a two-step process involving (i) adsorption of PDDA onto MLG and (ii) complexation of PDDA/PFO A photograph of MLG-PP obtained as an aqueous gum is also show in the image inset. Part of the scheme was adapted from ref. 28.

freestanding films. Such a process was carried out by simply pouring the methanol/MLG-PP composite dispersion into a large surface area tray and allowing slow solvent evaporation. Film formation occurred on the base of the tray, which could be peeled off from the bottom. Images of these steps are provided in Fig. S1 in the ESI. $\dagger$

A small section of the film was cut away to produce the freestanding composite film of MLG-PP (Fig. 2) with approximate dimensions of $23.5 \mathrm{~cm} \times 16.0 \mathrm{~cm}$. As can be seen, the film obtained was thin and flexible and could be bent with no apparent visible damage observed. The film had an average thickness of 131 microns as determined by SEM imaging (vide infra). This flexible nature was attributed to the presence of PDDA-PFO composite. The MLG-PP films were found to be sufficiently robust to be utilised and did not require them to be coated on any substrate. With these in hand, the next step of our investigation focused on the analysis and application of the new material.

X-Ray Photoelectron Spectroscopy (XPS) was utilised to analyse the surface elements present within the MLG-PP composite. The quantitative data revealed that the surface contained the elements carbon (55.1\%), fluorine (35.4\%), oxygen

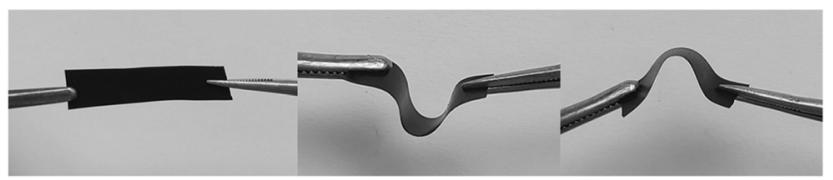

Fig. 2 Photographs of the MLG-PP film between two tweezers highlighting the flexible nature of the material.
(4.7\%), nitrogen (3.4\%), chlorine (1.1\%) and sodium (0.3\%). These values were consistent with the incorporation of both PDDA and PFO moieties onto the graphitic surface with residual quantities of salt from the synthetic method remaining within the structure. The deconvoluted C 1s spectrum for MLGPP (see Fig. S2 in ESI $\dagger$ ) confirmed the expected environments for the MLG, PDDA and PFO constituents of the composite. This was most evident by the presence of the perfluorinated carbon chains $\left(\mathrm{CF}_{2}\right.$ and $\mathrm{CF}_{3}$ at 291.5 and $\left.293.8 \mathrm{eV}\right)$ on the PFO units and the more characteristic of the two peaks expected for the PDDA units at $286.0 \mathrm{eV}$ which corresponds to the $\mathrm{sp}^{3}$ carbon functional groups directly attached to the positively charged nitrogen centre.

\section{Assessment of MLG-PP films in allowing oil/water passage}

The MLG-PP films were subsequently tested for their abilities to act as membranes for oil-water separation. To test how well they enabled water passage whilst maintaining simultaneous oleophobic behaviour, a burette was filled with silicone oil dyed with "oil red O", and deionised (DI) water dyed with "brilliant blue R". The liquids were then deposited drop-by-drop (approximately 1 drop per second) onto the films (Fig. 3). Due to its higher density, water was added first. In the first instance, $5 \mathrm{~mL}$ of DI water was added onto the MLG-PP film. The water droplets wetted the surface completely after around 30 seconds. Despite the wetting process occurring easily, the passage of water through the film was very slow. This is likely to be due to the thickness of the film. In some cases, it took up to 2.5 hours for the complete penetration and passage of water through the film. The image, Fig. 3d(i) shows that after 2 hours, the film became saturated with water and a droplet formed at the bottom of the film. This was not released into the beaker until a further 30 minutes had elapsed, as shown in Fig. 3d(ii)-(vii). Upon deposition of $5 \mathrm{~mL}$ silicone oil, it was found that no oil penetration occurred, and it remained on the surface of the film

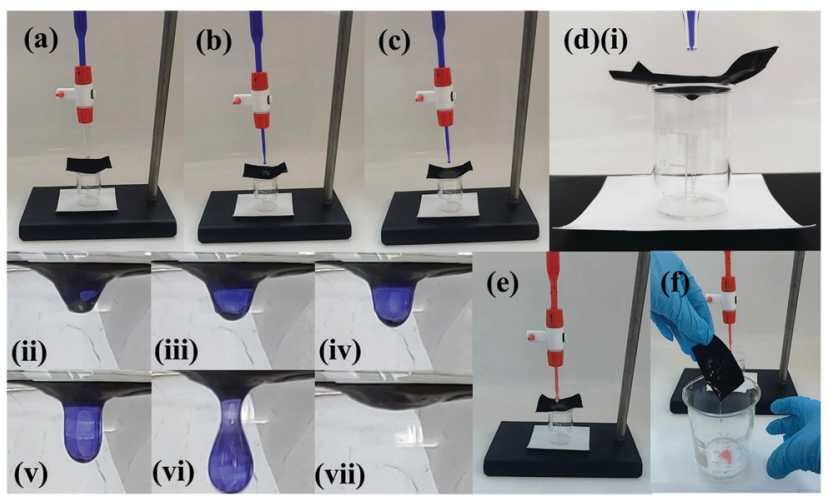

Fig. 3 (a) MLG-PP film prior to water/oil deposition; (b) water droplet formation on MLG-PP film instantly after deposition; (c) 30 seconds after water deposition, water droplets completely wet the surface of the film and slowly begin to penetrate the film (d) (i) water droplet remains as a droplet on the bottom of the film, 2 hours after deposition (d) (ii)-(vii) water droplet is released from the film after 2.5 hours (e) oil droplets instantly after deposition and ( $f$ ) oil drops poured off the film after 3 weeks. 
(Fig. 3e). The silicone oil remained on the surface of the film even after extended periods of time (at least three weeks). The silicone oil could then be poured off the surface easily to ensure its recovery, as shown in Fig. 3f. There was no evidence at any point in our investigations where the oil penetrated the film. The separation tests provided evidence that the MLG-PP film displayed oleophobic characteristics. Even though the passage of water through this film was slow, Fig. 3f demonstrates 100\% efficiency in regard to its oil/ water separation as no water was present in the liquid poured from the top of the film. $\neq \S$

\section{Contact angle measurements}

To gain an understanding of the extent of oleophobic behaviour shown by the composite film, contact angle measurements were carried out using silicone oil, hexadecane and Jet A-1 fuel. Contact angle analysis suggested that the MLG-PP films exhibited particularly high contact angles with these three liquids at $126.4^{\circ}, 111.9^{\circ}$ and $105.9^{\circ}$ confirming their oleophobic behaviour (see Fig. S5, ESI $\dagger$ for contact angle images). ${ }^{29}$ The contact angles decreased for oils in the order of hexadecane $>$ Jet A-1 fuel $>$ silicone oil. These correlate with the reported surface tension values for these liquids. ${ }^{30,31}$

In the case of water, it was found to wet the MLG-PP film surfaces instantly which indicates a negligible contact angle, thus confirming the hydrophilic nature of the film. This is likely due to the presence of the perfluorinated carbon chains extending outwards from the surface of the material providing a sufficiently high polar component and sufficiently low dispersive component of its surface tension. The combination, thus provides effective oleophobic/hydrophilic behaviour similar to that found in other investigations. ${ }^{1,10,11,13-20}$ When water is deposited on the surface, it also encounters fluorocarbon chains first. However, there is a stronger interaction between water and the polar $\mathrm{C}-\mathrm{F}$ bonds. ${ }^{20,32}$ During this dynamic process, water is driven towards the more hydrophilic groups of the composite allowing its passage through the pores of the composite.

\section{Scanning Electron Microscopy (SEM) and Atomic Force Microscopy (AFM) analysis}

SEM analysis was conducted to gain an insight to the structure and morphology of the MLG-PP film (Fig. 4 and Fig. S3, S4 in the ESI $\dagger)$. Fig. S4 (ESI $\dagger$ ) shows a representative image of the

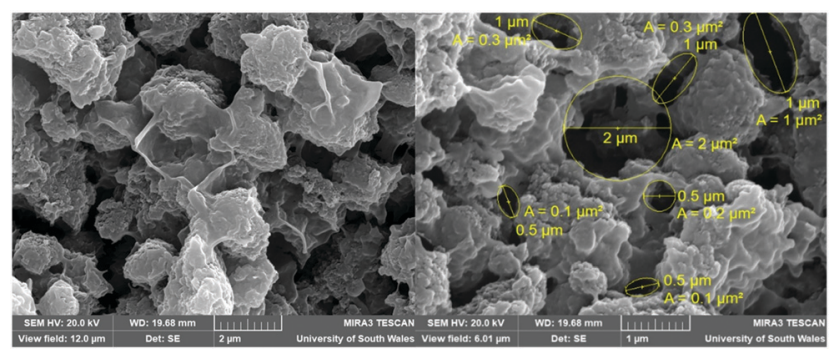

Fig. 4 Representative SEM images of MLG-PP film at higher magnifications. Pore size measurements have been added to the image on the right. side aspect of the film allowing an approximate film thickness to be determined. It was found that the film contained aggregates with average dimensions $<10 \mu \mathrm{m}$ in size with a small number of larger aggregates having widths of up to $100 \mu \mathrm{m}$. This was attributed to the synthetic conditions used for the preparation of the films, which involved a sonication step. In this step, a MLG-PP/methanol dispersion was formed, resulting in the exfoliation of large aggregates into smaller ones. The imaging showed that some aggregates are cross-linked with one another, presumably by PDDA/PFO complex, as shown in Fig. 4 (left). It was also noticeable that the aggregates contained a scaly, rippling texture. The arrangement of these aggregates and the presence of cross-linking resulted in the formation of pores throughout the MLG-PP film. This becomes more apparent at higher magnifications. Approximations were made to estimate the widths and areas associated with these pores as outlined within Fig. 4 (right). It was observed that the pores exhibited approximate widths of $0.5,1.0$ and $2.0 \mu \mathrm{m}$ with areas corresponding to $0.1,0.2,0.3,1.0$ and $2.0 \mu \mathrm{m}^{2}$. These pores were observable over twenty different SEM images taken at different positions across the film. The SEM analysis suggests that there is a network of pores which continue throughout the MLG-PP film and that these pores provide the most facile pathway of liquid through the material.

The morphology of MLG-PP film was analysed by AFM (Fig. 5). The film exhibited a textured and rough surface. The images show that the roughness components range extensively between approximately $10 \mathrm{~nm}$ to $5 \mu \mathrm{m}$. The coloured contrast of the images enables the height variations of the film to be observed where the lighter areas depict regions where the film protrudes from the surface and darker regions depict holes within the film. Further analysis of the holes is observed within Fig. $5 \mathrm{~b}$ and c. The representative line profiles across sections of the surface reveal relatively large drops in height within these holes, for example 37, 307, 240 and $276 \mathrm{~nm}$. It is believed that the material's roughness plays an important role in the oleophobic-hydrophilic and porous nature of these films. Previous studies have utilised $\mathrm{SiO}_{2}$ for this purpose. ${ }^{20,27}$ In our case, the nature of the plasma-exfoliated multi-layer graphitic material provides these properties through defects and partial oxidation of the layers.

\section{Experimental}

\section{Synthesis of MLG-PP Composite}

A PDDA aqueous solution (7.2 $\mathrm{mL}, 20 \mathrm{wt} \%$ in water) was added to MLG $(0.5604 \mathrm{~g})$ and placed in a beaker within an ultrasonic bath for 10 minutes. The resulting dispersion was stirred for a further 10 minutes. In a separate beaker, PFO (1.75 g) was dispersed in $10 \mathrm{~mL}$ DI water using an ultrasound bath for 10 minutes. This dispersion was slowly added to the MLG/ PDDA mixture over five minutes. Upon addition of PFO to MLG/ PDDA, a thick slurry formed immediately. The slurry was dispersed in DI water, then centrifuged and the supernatant decanted to remove any non-adsorbed material. This process 

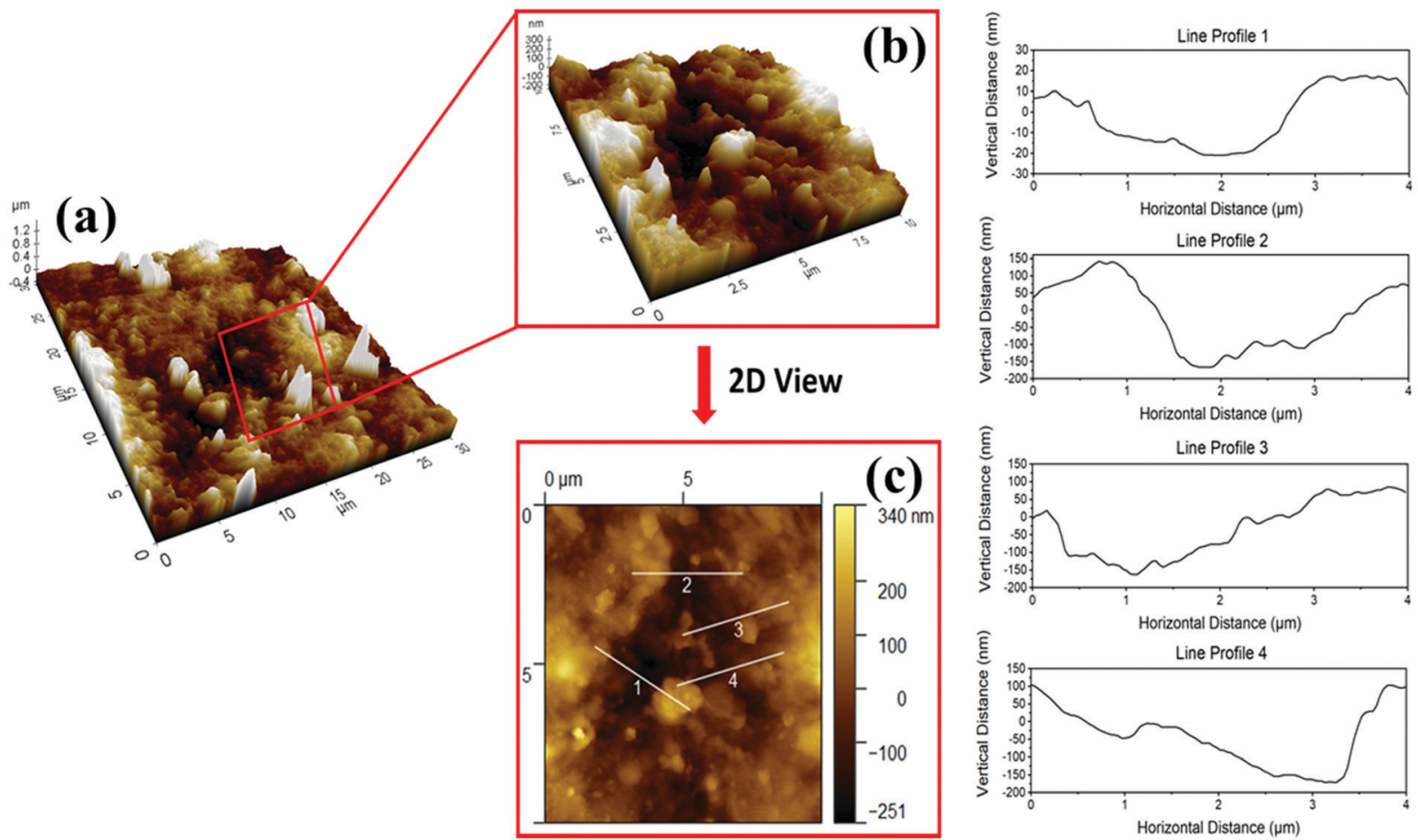

Fig. 5 AFM images of MLG-PP film depicting: (a) an AFM image of the surface of MLG-PP film, consisting of many aggregates; (b) a magnified image of (a); (c) a two-dimensional representation of image (b) containing line profiles 1-4, measured across four holes. The corresponding line profiles are shown on the right-hand column.

was repeated twice. The solid was then dried under vacuum for 12 hours to give the MLG-PP composite.

\section{Synthesis of MLG-PP Film}

To prepare a single MLG-PP film, $0.8 \mathrm{~g}$ of MLG-PP composite solid was dispersed in $35 \mathrm{~mL} \mathrm{MeOH}$ via the ultrasonic bath sonication for 15 minutes then poured into a single section of a large surface tray. This was left overnight for the solvent to evaporate slowly, providing a thin freestanding composite film denoted as MLG-PP film.

\section{Conclusions}

In summary, we have reported the development of a unique composite containing the polyelectrolyte/surfactant complex PDDA and PFO utilising multi-layered graphitic (MLG) material as the scaffold. The composite was used to prepare flexible freestanding MLG films by an easy drop-casting procedure. The films exhibited oleophobic behaviour to oils including silicone oil, hexadecane and Jet A-1 fuel. Oil contact angles up to $126.4^{\circ}$ were found for the MLG-PP films.

The films also show oleophobic/hydrophilic properties, and a preliminary investigation has demonstrated promise for applications in oil-water separations. It has been shown that water can pass through the material and oils are maintained at the surface. The microscopy imaging techniques reveal that channels do exist within the MLG-PP films which allow the passage of water through the films, albeit slowly. In order to enhance the transport of water through the material, these channels will need to be more open whilst at the same time maintaining the oleophobic characteristics. As such further investigations are currently under way exploring ways in which the durability and separation performances of these materials can be optimised. This will entail methodologies to enhance the rate of water passage through the film and ensuring more robust materials which allow for oil-spill clean-up on a practical scale and suggests potential within other separation applications.

\section{Conflicts of interest}

There are no conflicts to declare.

\section{Acknowledgements}

This work was funded by the European Social Fund (ESF) via the Welsh Government through a KESS2 PhD studentship (R. L. M). It was co-sponsored by Perpetuus Carbon Technologies. The company is also thanked for providing the raw MLG material. We are also grateful to the Harwell XPS EPSRC National Facility for carrying out the XPS data collections.

\section{References}

† Two additional MLG-PP films were prepared and investigated; one thinner and one thicker than the original. The thinner one had an average thickness of $27 \mu \mathrm{m}$ and the thicker one had an average thickness of $358 \mu \mathrm{m}$. The thinner one allowed complete water passage after 1 hour. It was, however, too weak in strength and was prone to 
tearing. The thicker one did not allow water passage and thus was unsuitable for the intended separation application. As such there is a correlation between thickness of the film and the rate of passage of water. This will be explored in further detail as part of our ongoing investigations.

$\S$ The integrity of the MLG-PP film to various solvents was investigated. A small sample of MLG-PP film was deposited into water, hexane, DCM, diethyl ether and acetone in separate flasks. These were vigorously stirred using a magnetic stirrer bar for 5 minutes. No visible fragmentation or changes in the films were observed during this time.

1 Y. Wang and X. Gong, J. Mater. Chem. A, 2017, 5, 3759-3773.

2 D. W. Wei, H. Wei, A. C. Gauthier, J. Song, Y. Jin and H. Xiao, J. Bioresour. Bioprod., 2020, 5, 1-15.

3 F. Guan, Z. Song, F. Xin, H. Wang, D. Yu, G. Li and W. Liu, J. Bioresour. Bioprod., 2020, 5, 37-43.

4 J. Zhang, L. Zhang and X. Gong, Langmuir, 2021, 37, 6042-6051.

5 L. Zhong, H. Tao and X. Gong, Langmuir, 2021, 37, 6765-6775.

6 R. L. McLaren, C. J. Laycock, D. J. Morgan and G. R. Owen, New J. Chem., 2020, 44, 19144-19154.

7 R. L. McLaren, C. J. Laycock, E. Brousseau and G. R. Owen, New J. Chem., 2021, 45, 12071-12080.

8 I. Walters and D. Walters, EU Pat., Perpetuus Research \& Development Limited, EP3129323B1, 2019.

9 J. Sun, H. Bi, S. Su, H. Jia, X. Xie and L. Sun, J. Membr. Sci., 2018, 553, 131-138.

10 S. J. Hutton, J. M. Crowther and J. P. S. Badyal, Chem. Mater., 2000, 12, 2282-2286.

11 F. Li, Z. Wang, S. Huang, Y. Pan and X. Zhao, Adv. Funct. Mater., 2018, 28, 1706867.

12 F. R. Sultanov, C. Daulbayev, B. Bakbolat and Z. A. Mansurov, Eurasian Chem.-Technol. J., 2018, 20, 195.

13 R. A. Lampitt, J. M. Crowther and J. P. S. Badyal, J. Phys. Chem. B, 2000, 104, 10329-10331.

14 J. Yang, Z. Zhang, X. Xu, X. Zhu, X. Men and X. Zhou, J. Mater. Chem., 2012, 22, 2834.
15 P. S. Brown, O. D. L. A. Atkinson and J. P. S. Badyal, ACS Appl. Mater. Interfaces, 2014, 6, 7504-7511.

16 P. S. Brown and B. Bhushan, Sci. Rep., 2015, 5, 14030.

17 P. S. Brown and B. Bhushan, Sci. Rep., 2015, 5, 8701.

18 H. Yoon, S.-H. Na, J.-Y. Choi, S. S. Latthe, M. T. Swihart, S. S. Al-Deyab and S. S. Yoon, Langmuir, 2014, 30, 11761-11769.

19 J. Yang, L. Yin, H. Tang, H. Song, X. Gao, K. Liang and C. Li, Chem. Eng. J., 2015, 268, 245-250.

20 J. Li, L. Yang, H. Liu, G. Li, R. Li, Y. Cao and H. Zeng, ACS Appl. Mater. Interfaces, 2020, 12, 45266-45273.

21 F. M. Fowkes, Ind. Eng. Chem., 1964, 56, 40-52.

22 F. M. Fowkes, Y. C. Huang, B. A. Shah, M. J. Kulp and T. B. Lloyd, Colloids Surf., 1988, 29, 243-261.

23 D. K. Owens and R. C. Wendt, J. Appl. Polym. Sci., 1969, 13, 1741-1747.

24 C.-T. Hsieh, J.-M. Chen, R.-R. Kuo, T.-S. Lin and C.-F. Wu, Appl. Surf. Sci., 2005, 240, 318-326.

25 T. Leo Liu and C.-J. Kim, Science, 2014, 346, 1096-1100.

26 H. C. Kang and A. M. Jacobi, Langmuir, 2011, 27, 14910-14918.

27 X. Du, X. Li and J. He, ACS Appl. Mater. Interfaces, 2010, 2, 2365-2372.

28 H. Dautzenberg, E. Görnitz and W. Jaeger, Macromol. Chem. Phys., 1998, 199, 1561-1571.

29 J. Yong, F. Chen, Q. Yang, J. Huo and X. Hou, Chem. Soc. Rev., 2017, 46, 4168-4217.

30 J. B. Boreyko, G. Polizos, P. G. Datskos, S. A. Sarles and C. P. Collier, Proc. Natl. Acad. Sci. U. S. A., 2014, 111, 7588-7593.

31 J. T. Edwards, 55th AIAA Aerospace Sciences Meeting, American Institute of Aeronautics and Astronautics, Reston, Virginia, 2017, pp. 1-58.

32 J. R. Robalo, L. M. Streacker, D. Mendes de Oliveira, P. Imhof, D. Ben-Amotz and A. V. Verde, J. Am. Chem. Soc., 2019, 141, 15856-15868. 